\title{
Performance Analysis of Passive UHF RFID Systems under Cascaded Fading Channels and Interference Effects
}

\author{
Abdelmoula Bekkali, Member, IEEE, Sicheng Zou, Student Member, IEEE, Abdullah Kadri, Member, IEEE, and \\ Richard Penty, Senior Member, IEEE
}

\begin{abstract}
In this paper, the performance of monostatic and bistatic passive ultrahigh-frequency radio-frequency identification (UHF RFID) systems under the effects of cascaded fading channels and interference is studied. The performance metric used is tag detection probability defined as probability that the instantaneous received power is higher than the receiver's sensitivity. A closed-form expression of the detection probability is derived using cascaded forward and backscatter fading channels and reader antennas orientation. Furthermore, the performance of passive RFID systems under reader-to-tag interference caused by both the desired RFID signal and multiple RFID interferers is analyzed, and the effect of constructive and destructive interferences is examined. In addition, the maximum reading range in ideal, multipath fading and interfering environments is presented. The obtained results are very useful for the design and optimization of passive RFID systems from RF point of view.
\end{abstract}

Index Terms-Passive UHF RFID, Detection Probability, Cascaded Fading Channels, Interference, Reliable Reading Range.

\section{INTRODUCTION}

$\mathbf{U}$ ltra high frequency radio frequency identification (UHF RFID) systems have gained attraction as an effective wireless technology for object identification. It has been widely adopted for applications like asset management, indoor localization, access control, and industrial automation [1]-[3]. RFID communication link is fundamentally different from that of conventional RF communication because it involves two distinct links: the power-up (Forward) link for powering passive RF tags, and the backscatter (Reverse) link for describing backscatter communication. Other ways in which RFID systems differ from conventional RF communication are some unique design factors that may create potential challenges for interference mitigation which include [4]: 1) the integrated circuits (ICs) embedded in RFID tags have limited tuning capability, and thus it is more susceptible to interference from RF signals, 2) the UHF Gen2 tags do not generate their own signal on a separate channel and they simply reflect the reader signal with the same frequency adding modulation to represent

Manuscript received March 16, 2014.

This work was made possible by NPRP grant NPRP4-726-2-272 from the Qatar National Research Fund (a member of Qatar Foundation). The statements made herein are solely the responsibility of the authors.

A. Bekkali and A. Kadri are with Qatar Mobility Innovation Center (QMIC), Doha, Qatar, e-mail: \{abdelmoulab, abdullahk\}@qmic.com

S. Zou and R. Penty are with Electrical Division, Department of Engineering, University of Cambridge, Cambridge, UK. e-mail: \{zs271, rvp11\}@cam.ac.uk their data, and 3) the reflected signal by the tag is many orders of magnitude weaker than the signals transmitted by the RFID reader. In general, there are two typical RFID system configurations based on RFID reader antennas implementation refereed to as monostatic and bistatic. For monostatic configuration, a single antenna is employed to simultaneously transmit the continuous wave $(\mathrm{CW})$ signal to power the tag as well as receive the backscattered signal from the tag. On the other hand, for bistatic configuration, the RFID reader uses two colocated antennas for separate transmit and receive [5].

The main factors influencing the reliability of a tag response include tag location and orientation, impedance mismatch between tag antenna and chip [6], multipath fading [7], communication blind spot [8], and interference (i.e. tag-to-tag, reader-to-tag and reader-to-reader interference). Furthermore, tag placement on a highly dielectric materials (i.e. liquids) or conductors (i.e. metal) can drastically change the properties of the tag antenna, and consequently reducing reading efficiency and shortening the reading range to the point of becoming completely unreadable at any distance in some cases [9]. Usually, these factors are beyond the control of the system user and therefore, for a maximum reliable reading range (i.e. $100 \%$ successful detection probability), proper conditions should be analyzed and defined before any implementation of the RFID system. The main performance metric of RFID system is the reading range or coverage that is defined as the maximum distance between the reader and the tag, where the radiation field from the reader is strong enough to power up the tag and the backscatter signal from the tag reaches the reader with sufficient power (i.e. with power above readers sensitivity threshold).

Although, many studies related to the passive UHF RFID system reading coverage improvement have been conducted, there are few analytical models that highlight the effects of the channel fading [5],[10]-[12], interference [13], [14] and readers antenna orientation [15], [16] on the interrogation zone reliability. In [5], the authors measured and compared the multipath channel fading for both single and multiple RFID antennas. The probability of successful tag detection is evaluated in [10] assuming that the RFID channel fading was modeled by Rician distribution. A statistical model suitable for bistatic and monostatic RFID configurations with multiple reader and tag antennas was presented in [11] where diversity gain was investigated by utilizing multiple tags. In [12], the interrogation zone with multiple transmit/receive antennas was 


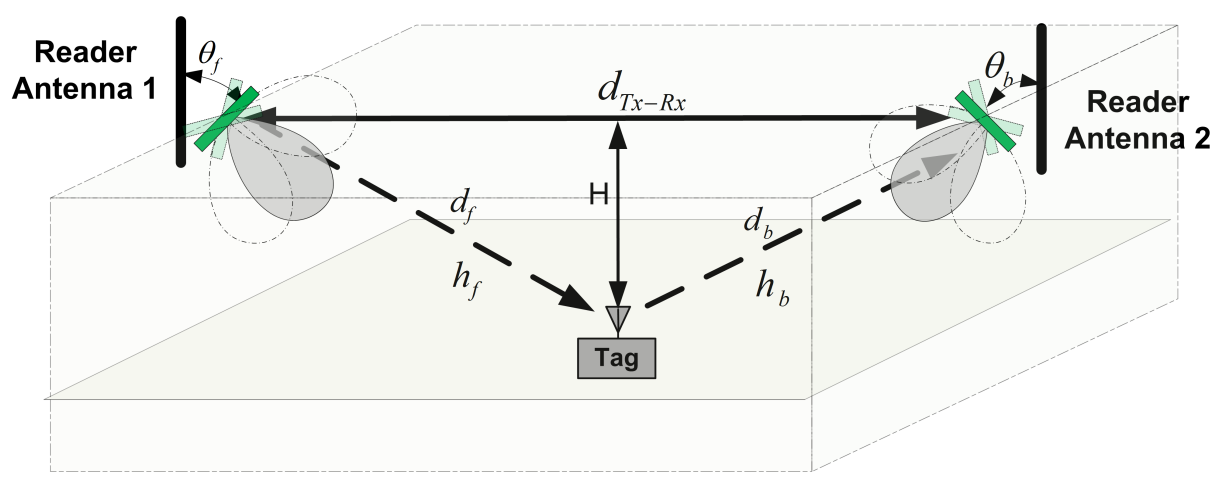

Fig. 1. Basic configuration of passive RFID system.

analyzed where the forward and backscattered channels are assumed to take the Nakagami- $m$ distribution. However, all these models calculate the interrogation range assuming omnidirectional antennas while poor tag orientation with respect to the reader antennas can result in an unfavorable link loss and thus drastically reduce the reading reliability. Other research efforts have analyzed the effect of the interference on the tag's detection [13], [14]. In [13], the authors investigated and analyzed the effect of the interference on the interrogation range reduction while in [14], RFID reader-to-reader interference was analyzed where a model to estimate the minimum distance between readers to achieve a desired probability of detection in real multipath environments was derived and compared to the ideal case. On the other hand, other research efforts have focused on modeling and analyzing the effect of the readers antenna orientation on the reading coverage [15], [16]. In [15], the authors introduced a multipath model taking into account the placement, orientation and polarization of the antenna. Using the proposed model, the optimization of the antennas position and tilt angle was carried out. A systematic formulation for the reading range of the reader-tag platform in the presence of multipath propagation, which is suitable for single-lobe directional antennas, was proposed in [16]. The reading region was approximated by an ellipsoid including reader antenna's location, while its axes depend on the halfpower beamwidth of the antenna. However, these models do not provide statistical analysis of the reliable interrogation range in the presence of fading channels.

In this paper, we model and analyse the effect of cascaded channel fading and readers antenna orientation on the passive RFID tags, in terms of detection probability defined as the probability that the instantaneous received power is higher than a specified RFID receiver sensitivity. We derive a closed-form expression for passive RFID detection probability taking into consideration the reader antennas orientations and cascaded forward and backscattered fading channel. We also study the performance of the passive RFID systems taking into account the reader antennas orientation and Rician fading channel for both desired RFID signal and multiple RFID interferers. Using the presented model, the effect of constructive and destructive (specifically tag jamming) interferences in passive RFID system is examined from the RF point of view. To the best of our knowledge, this is the first work that provides $3-\mathrm{D}$ system model and analysis of RFID system under cascaded channel fading and interference effects.

The paper is organized as follows. In Section II, the main expressions of passive RFID channel model are revised. A closed-form expression for passive RFID system detection probability is presented in Section III. Section IV introduces a closed-form expression for detection probability in the presence of multiple RFID interferers. In Section V, we derive the maximum reading range for both ideal, multipath fading and interfering environments. Simulation results are given in Section VI, where the effect of fading and interference on the reading coverage are studied. Finally, Section VII concludes the paper.

\section{Channel Modeling of Bistatic Passive RFID SYSTEM}

The operation of RFID system requires the communication between the RFID reader and the tag through two distinct links: the forward link and the backscatter link. The forward link (denoted by a subscript $f$ ), also called the reader-to-tag link, describes signal propagation from the reader's transmitting antenna to the RF tag, while the backscatter link (denoted by a subscript $b$ ), or the tag-to-reader link, describes signal propagation from the RF tag to the reader's receiving antenna. For a successful tag detection, two conditions must be realized: 1) the power received at the tag must be higher than the power-up threshold (i.e. tag sensitivity), and 2) the reader must be sensitive enough to detect correctly the backscattered modulation from the tag. Consequently, the reading range is usually limited by either forward or backscattered link pathlosses. The system is called forward-link limited (FLL) when the reading range is limited by the tag sensitivity $S_{T}$, in the other hand, the system is called backscattered link limited (BLL) when the reading range is limited by the reader sensitivity $S_{R}$. In a well-designed RFID system, reading range is limited by the forward link so that the overall system will naturally improve as tag sensitivity improves. However, this is found to be false and the RFID system range can be limited by the backscattered link as well [17].

The forward link power impinging on the tag antenna using reader directional antenna can be expressed as follows:

$$
P_{r, T}=\rho_{L} P_{t x} G_{T} G_{R}\left(d_{f}, H, \theta_{f}, \phi_{f}\right) L\left(d_{f}\right)\left|h_{f}\right|^{2},
$$


where $P_{t x}$ is the power transmitted by the RFID reader's antenna, $d_{f}$ is the reader to tag distance, $G_{R}\left(d_{f}, H, \theta_{f}, \phi_{f}\right)$ and $G_{T}$ are the gains of the RFID reader and tag antennas respectively, $h_{f}$ is the link fading coefficient, where its envelope can follow the Rician or Rayleigh distributions, and $\rho_{L}$ is the polarization loss factor (PLF) which reflects the loss due to the mismatch between the polarization of a transmitter antenna and the tag antenna. When readers have a circularpolarized antenna, the PLF is 0.5 (i.e. $3 \mathrm{dBm}$ loss) no matter what polarization the dipole tag antenna has [1]. $L\left(d_{f}\right)$ is the channel pathloss which can be modeled as the sum of reflected waves from ground, walls or other objects and given by:

$L\left(d_{f}\right)=\left(\frac{\lambda}{4 \pi d_{f}}\right)^{2}\left|1+\sum_{n=0}^{N} \Lambda_{n} \frac{d_{f}}{d_{n}} \exp \left(-j \frac{2 \pi\left(d_{f}-d_{n}\right)}{\lambda}\right)\right|^{2}$

where $d_{n}$ is the total distance of the $\mathrm{n}^{t h}$ reflected ray path and $N$ is the total number of reflections. The parameter $\Lambda_{n}$ is the Fresnel's reflection coefficient for the $\mathrm{n}^{\text {th }}$ wave and given by:

$$
\Lambda_{n}=\frac{\cos \theta_{n}-q \sqrt{\epsilon_{c}-\sin ^{2} \theta_{n}}}{\cos \theta_{n}+q \sqrt{\epsilon_{c}-\sin ^{2} \theta_{n}}}
$$

where $\epsilon_{c}$ is the complex permittivity of the ground, $\theta_{n}$ is the incident angle with the normal to ground, and $q$ is a polarization dependent factor that equals 1 for horizontal polarization and $\frac{1}{\epsilon_{c}}$ for vertical polarization [10]. In general, $\Lambda_{n}$ depends on the material properties, wave polarization, angle of incident and the frequency of the wave [18]. In a pure multipath environment, where many equal amplitude and uniformly distributed phase replicas of the transmitted signal arrive at the receiver, the short term fading envelope will have a Rayleigh probability density function (pdf) given by [19]:

$$
h_{\text {Ray }}(r)=\frac{r}{\sigma^{2}} \exp \left(-\frac{r^{2}}{2 \sigma^{2}}\right) \quad r \geq 0
$$

where $r$ is the envelope of the received voltage (i.e. $r^{2} / 2$ is the short term signal power) and $\sigma$ its root mean square (rms) value before envelope detection. However, when there is a lineof-sight (LOS), or at least a dominant specular component the short term fading envelope will have a Rician pdf given by:

$$
h_{\text {Rice }}(r)=\frac{r}{\sigma^{2}} \exp \left(-\frac{r^{2}+a^{2}}{2 \sigma^{2}}\right) I_{0}\left(\frac{r a}{\sigma^{2}}\right) \quad r \geq 0, \quad a \geq 0
$$

where $I_{0}(\bullet)$ is the modified Bessel function of the first kind and order zero, and $a$ denotes the peak amplitude of the dominant signal of the dominant component. It can be noticed that the Rayleigh distribution is a special case of the Rician distribution when $a=0$ (i.e. complete disappearance of the specular power). A commonly used notation for the dominant to multipath signal power ratio for the Rician distribution is $K=a^{2} /\left(2 \sigma^{2}\right)$. The parameter $K$ is then called Rician $K-$ Factor and when it is large, it indicates a strong dominant path. This type of multipath, i.e. Rician fading, presents more realistic environment in RFID communication. Reported Rician $K$-Factors for forward and backscatter channels lie between $-\infty \mathrm{dB}$ and $2.8 \mathrm{~dB}$ [7]. These numbers represent the $K$ factors of the individual reader-to-tag and tag-to-reader links, where it has been assumed that each link has the same
$K$-Factor.

Assuming the scenario illustrated in Fig. 1, and adopting the expression provided by [20], a modified directional gain of a patch antenna can be expressed as follows:

$G_{R}\left(d_{f}, H, \theta_{f}, \phi_{f}\right)=3.136\left[\tan \left(\alpha_{f}\right) \sin \left(\frac{\pi}{2} \cos \left(\alpha_{f}\right) \sin \left(\phi_{f}\right)\right)\right]^{2}$

where $\alpha_{f}=\theta_{f}+\arcsin \left(\frac{H}{d_{f}}\right) ; \theta_{f}$ and $\phi_{f}$ are the inclination and azimuthal angles of the patch antenna respectively. $H$ is the distance between tag location and its orthogonal projection on the reader plane $(x, y)$ when $\theta_{f}=0$. In the following and for the sake of simplicity of notations, we replace $G_{R}\left(d_{f, b}, H, \theta_{f, b}, \phi_{f, b}\right)$ by $G_{R}^{f, b}$.

If the power received by the tag is sufficient to operate the tag, a backscattered signal from the tag is received by the reader. Thus, for a bistatic RFID system, the total backscattered power $P_{r, R}$ received by the reader is given by:

$$
P_{r, R}=\tau \mu_{T} \rho_{L} P_{t x}\left|G_{T}\right|^{2} G_{R}^{f} G_{R}^{b} L\left(d_{f}\right) L\left(d_{b}\right)\left|h_{f}\right|^{2}|\Gamma|^{2}\left|h_{b}\right|^{2}
$$

where the subscript notations $f$ and $b$ are used to describe the forward and backscattered links respectively. The parameter $\mu_{T} \in[0,1]$ is the power transfer efficiency that quantifies how well the tag is impedance-matched to the antenna. The normalized coefficient $\tau$ quantifies the specific data encoding modulation details and it can be calculated using Power Density Distribution (PSD) of the tag's signal [13]. According to the EPCglobal C1G2 specifications [21], any tag in the interrogation zone of the reader can send back its information by reflecting the incoming continuous wave using either FM0 or Miller subcarrier (Miller-2, Miller-4 or Miller-8) encoding schemes and Amplitude Shift Keying (ASK) modulation. The parameter $\Gamma=\Gamma_{1}-\Gamma_{2}$ is the differential reflection coefficient of the tag, where $\Gamma_{1}$ and $\Gamma_{2}$ are the complex power wave reflection coefficients between tag antenna impedance $Z_{A}$ and chip impedance $Z_{1,2}$ in modulating states 1 and 2 and defined by the following equation:

$$
\Gamma_{1,2}=\frac{Z_{1,2}-Z_{A}^{*}}{Z_{1,2}+Z_{A}} .
$$

Usually, the parameter $\Gamma$ is defined as a function of tag's Radar Cross Section $\sigma_{R C S}$, tag antenna gain $G_{T}$, and the communication wavelength $\lambda$ and is given by:

$$
|\Gamma|^{2}=\frac{4 \pi \sigma_{R C S}}{\lambda^{2}\left|G_{T}\right|^{2}} .
$$

In the case of a monostatic RFID reader (i.e. $h_{f}=h_{b}=$ $h_{m}, d_{f}=d_{b}=d, \theta_{f}=\theta_{b}=\theta, \phi_{f}=\phi_{b}=\phi$ ), the total backscattered power received by the reader can be simplified to:

$$
P_{r, R}=\tau \mu_{T} \rho_{L} P_{t x}\left|G_{T} G_{R}(d, H, \theta, \phi) L(d)\right|^{2}|\Gamma|^{2}\left|h_{m}\right|^{4}
$$

\section{Passive RFID System Detection Probability}

The channel fading in RFID systems can often follow a cascaded Rician distribution resulting in deeper fades compared to the signal received by the RFID tag (forward link). In this paper, we consider a bistatic dislocated RFID system configuration, where it is commonly accepted to have both 
LOS and non-line-of-sight (NLOS) in the communication link [7]. Therefore, the short term fading envelope can be modeled by both Rician and Rayleigh distributions. If we denote the coefficient $h=h_{f} h_{b}$ as the cascaded channel fading, where $h_{f}$ and $h_{b}$ are the Rician-forward and Rician-backscattered link fading parameters respectively, the envelope of $h$ can follow a statistical distribution where its pdf is given by [22]:

$$
\begin{aligned}
p_{h}(r)= & \frac{r \exp \left(-\left(K_{f}+K_{b}\right)\right)}{\sigma_{f}^{2} \sigma_{b}^{2}} \sum_{i=0}^{\infty} \sum_{l=0}^{\infty} \frac{1}{(i ! l !)^{2}}\left(\frac{r K_{f}}{2 \sigma_{f}^{2}}\right)^{i} \\
& \times\left(\frac{r K_{b}}{2 \sigma_{b}^{2}}\right)^{l}\left(\frac{\sigma_{f}}{\sigma_{b}}\right)^{i-l} \mathbf{K}_{i-l}\left(\frac{r}{\sigma_{f} \sigma_{b}}\right), r \geq 0 .
\end{aligned}
$$

where $K_{f}$ and $K_{b}$ are the Rician factors for both forward and backscattered links respectively, $\mathbf{K}_{\nu}$ is the modified Bessel function of the second kind with order $\nu$, and $\sigma_{f}$ and $\sigma_{b}$ are the rms values of the received voltage signals before envelope detection for both forward and backscattered links respectively.

The passive RFID system detection probability $\mathcal{D}$ is defined as the probability that the instantaneous received power is higher than the specified reader's antenna sensitivity $S_{R}$ and expressed as follows:

$$
\begin{aligned}
\mathcal{D} & =\operatorname{Prob}\left(P_{r, R} \geq S_{R}\right) \\
& =1-\operatorname{Prob}\left(P_{r, R} \leq S_{R}\right)
\end{aligned}
$$

Let's denote $A_{t h}$ as $\sqrt{S_{R} / P_{r, R}^{(0)}}$, where $P_{r, R}^{(0)}$ is defined as follows:

$$
P_{r, R}^{(0)}=\tau \mu_{T} \rho_{L} P_{T x}\left|G_{T}\right|^{2} G_{R}^{f} G_{R}^{b} L\left(d_{f}\right) L\left(d_{b}\right)|\Gamma|^{2}
$$

The average received power $\overline{P_{r, R}}$ is given by:

$$
\overline{P_{r, R}}=P_{r, R}^{(0)}\left(2 \sigma_{f} \sigma_{b}\right)^{2}\left(K_{f}+1\right)\left(K_{b}+1\right),
$$

thus,

$$
A_{t h}=\sigma_{f} \sigma_{b} \times \sqrt{\frac{4\left(K_{f}+1\right)\left(K_{b}+1\right) S_{R}}{\overline{P_{r, R}}}}
$$

Therefore, Eq.12 can be rewritten as:

$$
\begin{aligned}
\mathcal{D}=1 & -\int_{0}^{A_{t h}} p_{h}(r) d r \\
=1 & -\frac{\exp \left(-\left(K_{f}+K_{b}\right)\right)}{\sigma_{f}^{2} \sigma_{b}^{2}} \\
& \times \sum_{i=0}^{\infty} \sum_{l=0}^{\infty} \frac{1}{(i ! l !)^{2}}\left(\frac{K_{f}}{2 \sigma_{f}^{2}}\right)^{i}\left(\frac{K_{b}}{2 \sigma_{b}^{2}}\right)^{l}\left(\frac{\sigma_{f}}{\sigma_{b}}\right)^{i-l} \\
& \times \int_{0}^{A_{t h}} r^{i+l+1} \mathbf{K}_{i-l}\left(\frac{r}{\sigma_{f} \sigma_{b}}\right) d r .
\end{aligned}
$$

Applying the integration by substitution technique and after some manipulations, Eq.16 can be written as follows:

$$
\begin{aligned}
\mathcal{D}=1 & -2 \exp \left(-\left(K_{f}+K_{b}\right)\right) \sum_{i=0}^{\infty} \sum_{l=0}^{\infty} \frac{1}{(i ! l !)^{2}} \\
& \times\left(K_{f}\right)^{i}\left(K_{b}\right)^{l}\left(\frac{\left(K_{f}+1\right)\left(K_{b}+1\right) S_{R}}{\overline{P_{r, R}}}\right)^{\frac{i+l}{2}+1} \\
& \times \int_{0}^{1} r^{\frac{i+l}{2}} \mathbf{K}_{i-l}\left(\frac{A_{t h} \sqrt{r}}{\sigma_{f} \sigma_{b}}\right) d r
\end{aligned}
$$

Using the Meijer G-function ${ }^{1}$ properties ([23] p.1024, Eq.9.304.5) and solving the integral in Eq.17 ([23] p.682, Eq.6.592.2), a closed-form expression of the detection probability $\mathcal{D}$ can be expressed in terms of Meijer G-function $\mathbf{G}_{p, q}^{m, n}$, the average received power $\overline{P_{r, R}}$, the forward and backscattered link Rician $K$-factors and the RFID reader antenna sensitivity $S_{R}$ as shown in Eq. 18 .

In the case of cascaded Rayleigh and Rician fading (i.e. $K_{f \vee b}=0(-\infty \mathrm{dB})$, where the symbol $\vee$ is used to denote "or"), the reader detection probability can be simplified to:

$$
\begin{aligned}
\mathcal{D}_{\left(K_{f \vee b}=0\right)}=1 & -\exp \left(-K_{f \vee b}\right) \sum_{i=0}^{\infty} \frac{1}{(i !)^{2}}\left(K_{f \vee b}\right)^{i} \\
& \times \mathbf{G}_{1,3}^{2,1}\left(\frac{\left(K_{f \vee b}+1\right) S_{R}}{\overline{P_{r, R}}} \mid \begin{array}{c}
1 \\
i+1,1,0
\end{array}\right) .
\end{aligned}
$$

If the case of cascaded Rayleigh fading (i.e. $K_{f}=K_{b}=0$ ) is considered, then the detection probability can be simplified to:

$$
\mathcal{D}_{\left(K_{f}=K_{b}=0\right)}=1-\mathbf{G}_{1,3}^{2,1}\left(\begin{array}{c|c}
\overline{S_{R}} & \begin{array}{c}
1 \\
\overline{P_{r, R}}
\end{array} \\
1,1,0
\end{array}\right) .
$$

\section{INTERFERENCE IN PASSIVE RFID SYSTEM}

\section{A. Background}

The problem of interference in RFID system has been studied in the EPCglobal C1G2 standards for UHF readers [21] and in ETSI-302 - 208 which is the European regulation governing the operation of RFID readers [24]. However, dense reader mode introduced by EPCglobal C1G2 can not a cure all the limitations of today's passive RFID technology and both regulations are not entirely friendly for dense RFID reader deployment. In some cases, it is not possible to have a feasible RFID system while adhering to these regulations. There are two main interference types in RFID system:

1) Reader-to-Reader $(R 2 R)$ Interference: The R2R interference occurs when the signal from a neighboring reader interferes with tag responses being received at another reader. In Fig.2, the weak tag reply signal reaching Reader-1 from the tag can easily get distorted by the signal from Reader- 2 . But the extreme weakness of tag reply for passive tags means that a high degree of transmitter signal filtering is required. The $\mathrm{R} 2 \mathrm{R}$ interference does not only result in a reduction of over

\footnotetext{
${ }^{1}$ Meijer G-function [[23] p.1022 Eq.9.301], is a standard built-in function in most well known mathematical software packages such as Mathematica and Maple.
} 


$$
\mathcal{D}=1-\exp \left(-\left(K_{f}+K_{b}\right)\right) \sum_{i=0}^{\infty} \sum_{l=0}^{\infty} \frac{1}{(i ! l !)^{2}} \times\left(K_{f}\right)^{i}\left(K_{b}\right)^{l} \times \mathbf{G}_{1,3}^{2,1}\left(\frac{\left(K_{f}+1\right)\left(K_{b}+1\right) S_{R}}{\overline{P_{r, R}}} \mid \begin{array}{c}
1 \\
i+1, l+1,0
\end{array}\right)
$$

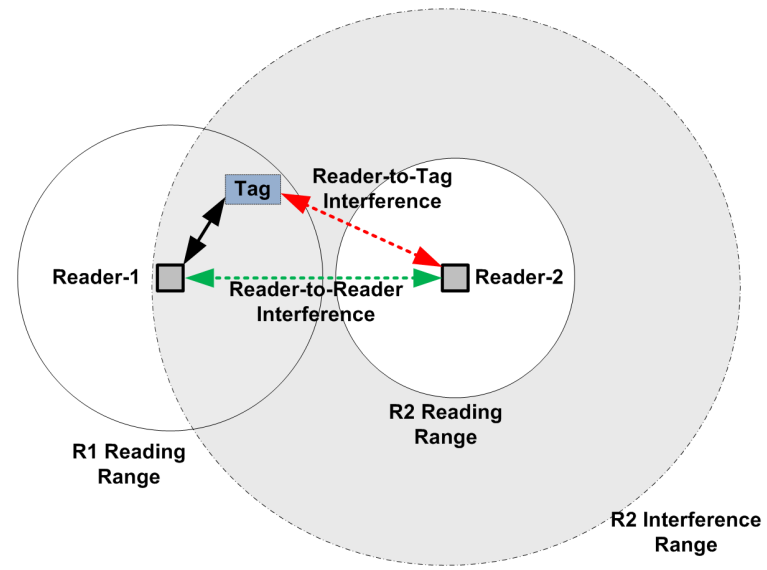

Fig. 2. Interference in RFID System.

all detection probability, but also it aggravates other problems such as increasing bandwidth usage and causing security risks. Mitigating factors for R2R interference include using welldesigned readers, proper selection of reader mode, channeluse randomization, shielding the reader, and the appropriate selection of reader antennas for the application [25],[26].

2) Reader-to-Tag (R2T) Interference: The $\mathrm{R} 2 \mathrm{~T}$ interference occurs when multiple readers simultaneously try to read a tag which is located within their reading ranges. Although a tag can listen to any reader in its vicinity, it can only reply to one reader (with stronger signal than other readers) at a time. In this situation, the tag might be unable to respond to any reader at all [27]. For example as shown in Fig.2, between any two readers, there is an area between them where a tag cannot communicate with either reader if both readers are transmitting. This phenomenon is known as tag jamming and the regions where tags are jammed can be seen as dead zones.

\section{B. Detection Probability in the Presence of Multiple RFID Interferers}

Spatial diversity with multiple antennas or phased array directional antenna can be good approaches for mitigating dead zones and thus increase the reading range reliability. However, this diversity can cause constructive interference which amplifies the received signal at tags location as well as destructive interference (tag jamming) which can result in deep fading. Constructive interference occurs whenever waves come together so that they are in phase with each other. This means that their oscillations at a given point are in the same direction, the resulting amplitude at that point can be much larger than the amplitude of an individual wave. On the other hand, destructive interference occurs when two waves having the same amplitude and opposite phases, neutralize each other. In multi-reader or multiple antennas scenarios, the total electric field outside the tag antenna will be the sum of the vectorial incident fields from $M$ transmitting antennas with the direction $\overrightarrow{i_{m}}$ and transmitter phase $\varphi_{m}$ and can be expressed as follows:

$$
P_{r, t o t a l}=30\left(\frac{4 \pi}{\lambda}\right)^{2}\left|\sum_{m=1}^{M} \sqrt{P_{r, m}} e^{j\left(\varphi_{m}-\frac{2 \pi d_{m}}{\lambda}\right)} \overrightarrow{i_{m}}\right|^{2}
$$

where $P_{r, m}$ is the received power from the $\mathrm{m}^{\text {th }}$ antenna defined by Eq.1.

It is well known that the for a single RFID reader with diversity antennas, it simply applies time division multiplexing techniques to prevent interference occurring between signals from different antennas. However, when multiple readers are present in the same working environment, signal from one reader may reach others and can cause R2T interference.

In this section, we will discuss the R2T interference scenario where the passive RFID is assumed to operates in a forwardlink limited regime (i.e. the read range is limited by the ability to deliver power to the tag). The detection probability $\mathcal{D}_{\mathcal{I}}$ will be used as performance metric of RFID system under interference effect and thus it will be defined with respect to a certain signal-to-interference (SIR) ratio, which can be considered as the minimum level of the tag signal in which the tag can detect and resolve. An adaptive closed-form expression from [28], is used to evaluate the detection probability for a desired reader signal received from a RFID tag in the presence of single or multiple interfering signals with Rician or Rayleigh distributed fading, and is given by :

$$
\begin{aligned}
{[h] \quad } & \mathcal{D}_{\mathcal{I}}\left(K_{0}, K_{I}, N_{I}, \mathrm{SIR}_{\mathrm{th}}\right) \\
= & 1-Q\left(\sqrt{\frac{2 N_{I} K_{I} \mathrm{SIR}_{\mathrm{th}}}{b_{1}+\mathrm{SIR}_{\mathrm{th}}}} ; \sqrt{\frac{2 K_{0} b_{1}}{b_{1}+\mathrm{SIR}_{\mathrm{th}}}}\right) \\
+ & \exp \left(-\frac{N_{I} K_{I} \mathrm{SIR}_{\mathrm{th}}+K_{0} b_{1}}{b_{1}+\mathrm{SIR}_{\mathrm{th}}}\right) \\
\times & \sum_{n=0}^{N_{I}-1}\left(\frac{K_{0} \mathrm{SIR}_{\mathrm{th}}}{N_{I} K_{I} b_{1}}\right)^{\frac{n}{2}} I_{n}\left(\sqrt{\frac{4 N_{I} K_{0} K_{I} b_{1} \mathrm{SIR}_{\mathrm{th}}}{b_{1}+\mathrm{SIR}_{\mathrm{th}}}}\right) \\
\times & \left\{\left(1+\frac{b_{1}}{\mathrm{SIR}_{\mathrm{th}}}\right)^{-N_{I}} \sum_{k=n}^{N_{I}-1}\left(\begin{array}{c}
N_{I} \\
k-n
\end{array}\right)\left(\frac{b_{1}}{\mathrm{SIR}_{\mathrm{th}}}\right)^{k}-\delta_{n 0}\right\} \\
& (n \geq 0),
\end{aligned}
$$

where $Q$ is the Marcum's function, $I_{m}(\bullet)$ is the modified Bessel function of the first kind and order $m, N_{I}$ is the number of interferers, $\delta_{n 0}$ is Kronecker delta, $\mathrm{SIR}_{\text {th }}$ is the signal-tointerference protection ratio and $K_{0}, K_{I}$ are the Rician Kfactor for the desired reader signal and the interfering reader, respectively. The parameter $b_{1}$ can be written as follows:

$$
b_{1}=\frac{K_{I}+1}{K_{0}+1} \mathrm{SIR},
$$

and

$$
\mathrm{SIR}=\frac{P_{D}}{\sum_{i=1}^{N_{I}} P_{I, i}},
$$


where $P_{D}$ is the desired reader signal power, and $P_{I, i}$ is the $\mathrm{i}^{\text {th }}$ interfering reader signals power and both defined by Eq. 1 . In this equation, it is assumed that all signals are independent and all interferers have the same Rician factor $K=K_{I}$.

\section{MAXimum ReAding RANGe}

Reading range is one of the most significant criteria in evaluating the performance of RFID systems, especially UHF RFID systems. This is because a longer reading range may create more potential applications. In order to ensure reliable reading range, it is important to do some analysis to find out the methods for evaluating the reading range and its influencing factors. In this section, we discuss and derive the maximum reader interrogation range in ideal, multipath fading and interference environments.

In the forward link, to activate the tag, the received signal power at the tag should be higher than the tag sensitivity $S_{T}$. Tag sensitivity is the minimum signal power at the tag location needed to power up the tag. Recently, the typical tag sensitivity of UHF Gen2 RFID chips have been reported ranging from -11 to $-18 \mathrm{dBm}$. However, it must be emphasized that the nominal threshold may not be accomplished in the entire operating frequency band, due to the variation of the tag IC impedance with frequency [30]. From Eq.1 we have:

$$
\rho_{L} P_{t x} G_{T} G_{R}^{f} L\left(d_{f}\right)\left|h_{f}\right|^{2} \geq S_{T},
$$

which is considered as the forward link constraint. Thus,

$$
\left|h_{f}\right|^{2} L\left(d_{f}\right) \geq \frac{S_{T}}{\rho_{L} P_{t x} G_{T} G_{R}^{f}}
$$

Therefore, the lower bound of the forward link pathloss is given by:

$$
\left|h_{f}\right|^{2} L\left(d_{f}^{\max }\right)=\frac{S_{T}}{\rho_{L} P_{t x} G_{T} G_{R}^{f}}
$$

In the backscattered link, to successfully demodulate the backscattered signal, the received backscattered power should be higher than the reader sensitivity $S_{R}$. Using Eq.7, we get:

$$
\tau \mu_{T} \rho_{L} P_{T x}\left|G_{T}\right|^{2} G_{R}^{f} G_{R}^{b} L\left(d_{f}\right)|\Gamma|^{2} L\left(d_{b}\right)\left|h_{f} h_{b}\right|^{2} \geq S_{R},
$$

which is considered as the backscattered link constraint. Therefore,

$$
\left|h_{f} h_{b}\right|^{2} L\left(d_{b}\right) \geq \frac{S_{R}}{\tau \mu_{T} \rho_{L} P_{t x}\left|G_{T}\right|^{2} G_{R}^{f} G_{R}^{b} L\left(d_{f}^{\max }\right)|\Gamma|^{2}}
$$

Hence, the lower bound of the backscattered link pathloss can be given by:

$$
\left|h_{f} h_{b}\right|^{2} L\left(d_{b}^{\max }\right)=\frac{S_{R}}{\tau \mu_{T} \rho_{L} P_{t x}\left|G_{T}\right|^{2} G_{R}^{f} G_{R}^{b} L\left(d_{f}^{\max }\right)|\Gamma|^{2}}
$$

Substituting Eq.27 into Eq.30, we have :

$$
\left|h_{b}\right|^{2} L\left(d_{b}^{\max }\right)=\frac{S_{R}}{\tau \mu_{T}|\Gamma|^{2}\left|G_{T}\right| G_{R}^{b} S_{T}}
$$

Next, we derive the maximum reader interrogation range in ideal, multipath fading and interference environments.

\section{A. Ideal Environment}

In the case of free space scenario, the path loss model is given by:

$$
L(d)=\left(\frac{\lambda}{4 \pi d}\right)^{2}
$$

Substituting Eq.32 into Eq.27 and Eq.31, we have :

$$
d_{f}^{\max }=\frac{\lambda}{4 \pi} \sqrt{\frac{\rho_{L} P_{T x} G_{T} G_{R}^{f}}{S_{T}}}
$$

and

$$
d_{b}^{\max }=\frac{\lambda}{4 \pi} \sqrt{\frac{\tau \mu_{T} S_{T} G_{T} G_{R}^{b}|\Gamma|^{2}}{S_{R}}}
$$

If we denote by $\delta=d_{f}^{\max } / d_{b}^{\max }$, then we have:

$$
\delta=\alpha \sqrt{\frac{P_{t x} S_{R}}{S_{T}^{2}}}
$$

where:

$$
\alpha=\rho_{L} G_{R}^{f} / \tau \mu_{T}|\Gamma|^{2} G_{R}^{b} .
$$

If $\delta \leq 1$, which means that $S_{T} \geq \alpha \sqrt{P_{t x} S_{R}}$, then the link is forward link limited. Otherwise, $S_{T}<\alpha \sqrt{P_{t x} S_{R}}$ and thus the link is backscattered link limited. Finally, the maximum reading range $d_{\text {Free Space }}^{\max }$ in ideal environment is given by:

$$
d_{\text {Free Space }}^{\max }= \begin{cases}d_{f}^{\max } & \text { when } S_{T} \geq \alpha \sqrt{P_{T x} S_{R}} \\ d_{b}^{\max } & \text { when } S_{T} \leq \alpha \sqrt{P_{T x} S_{R}}\end{cases}
$$

It should be noted that, as shown from the Eq.35, when the tag sensitivity $S_{T}$ continues to improve, it will increase faster compared to the quantity $\sqrt{P_{T x} S_{R}}$ and thus the link will become BLL.

\section{B. Multipath Fading Environment}

Most RFID systems are not deployed in free space but in indoor environment consisting of many obstacles in the signal propagating path. To evaluate the maximum interrogation range under fading channels, we define reliable interrogation range, as the maximum distance for a given detection probability $\mathcal{D}_{t h}$ and can be represented by:

$$
d_{\max } @ \mathcal{D}_{t h}=\underset{0 \leq d \leq d_{\text {free Space }}^{\max }}{\operatorname{argmax}} \mathcal{D}(d) \geq \mathcal{D}_{t h}
$$

where $\mathcal{D}(d)$ is the detection probability defined by Eq.18 and $d_{\mathrm{Free}}^{\max }$ Space is the maximum free space distance defined by Eq.37. For instance, without detection probability threshold constraint (i.e. $\mathcal{D}_{t h}=0$ ), the maximum distance $d_{\max }$ can simply be obtained when $S_{R}=\overline{P_{r, R}}$. However, at this distance and using the Eq. 20 the detection probability for the case of cascaded Rayleigh fading is about $28 \%$ only.

\section{Interference Environment}

Similar to the case of multipath fading environment, when the RFID system is subject to multiple interferes, the maximum interrogation range can be defined as the maximum 
TABLE I

PASSIVE RFID SySTEM PARAMETERS

\begin{tabular}{|l|l|}
\hline Parameter & Value \\
\hline \hline Operating Frequency & $865.7 \mathrm{MHz}$ \\
\hline Transmit Power $\left(P_{t x}\right)$ & $+33 \mathrm{dBm}$ EIRP \\
\hline Modulation Efficiency $(\tau)$ & 0.5 \\
\hline Polarization Loss Factor $\left(\rho_{L}\right)$ & 0.5 \\
\hline Power Transfer Efficiency $\left(\mu_{T}\right)$ & 1 \\
\hline Differential Reflection Coefficient $\left(\Gamma^{2}\right)[29]$ & 0.1 \\
\hline Fresnel's reflection coefficient $\Lambda_{n}$ & -1 \\
\hline Tag Ant Gain $\left(G_{T}\right)$ & $0 \mathrm{dBi}$ \\
\hline Tag Ant sensitivity $\left(T_{S}\right)$ & $-15 \mathrm{dBm}$ \\
\hline Tag Height $(H)$ & $1.5 \mathrm{~m}$ \\
\hline Reader Ant Sensitivity $\left(R_{S}\right)$ & $-75 \mathrm{dBm}$ \\
\hline Reader Ant Elevation Angle $(\theta)$ & $\pi / 4$ \\
\hline Antennas Separation Distance & $8 \mathrm{~m}$ \\
\hline
\end{tabular}

distance for a given detection probability $\mathcal{D}_{\mathcal{I}_{t h}}$ and is given by:

$$
d_{\max } @ \mathcal{D}_{\mathcal{I}_{t h}}=\underset{0 \leq d \leq d_{\text {Free Space }}^{\max }}{\operatorname{argmax}} \mathcal{D}_{\mathcal{I}}(d) \geq \mathcal{D}_{\mathcal{I}_{t h}}
$$

where $\mathcal{D}_{\mathcal{I}_{t h}}(d)$ is the detection probability defined by Eq.22 and $d_{\text {Free Space }}^{\max }$ is the maximum free space distance defined by Eq.37. It should be noted that the $\mathcal{D}_{\mathcal{I}}$ is calculated for the case of forward link, and thus $d_{\max } @ \mathcal{D}_{\mathcal{I} t h}$ can be considered as FLL maximum distance.

\section{Simulation Results}

In order to examine the impact of multipath fading and interference on the passive RFID system performance, we assume that we have two reader's antennas are placed on the ceiling of a (L: $8 \mathrm{~m} \times \mathrm{W}: 8 \mathrm{~m} \times \mathrm{H}: 3 \mathrm{~m})$ room size, and specifically located at positions $P_{1}(0,4,3)$ and $P_{2}(8,4,3)$. The reader antennas are facing each others as illustrated in Fig.1 and each reader is transmitting $+33 \mathrm{dBm}$ equivalent isotropically radiated power (EIRP). The overall system parameters are set in Table.I. The numerical results of tags detection probability and reader reading coverage are generated and studied based on equations Eq.7, Eq.18, Eq.19 and Eq.20.

Before presenting the numerical results, we define the reliable reading range as the maximum range with more than $90 \%$ detection probability. Figure 3 shows an example of the 3 -D view of reader coverage under multipath environment for the case of $K_{f}=K_{b}=0 \mathrm{~dB}$ and calculated based on bistatic two rays model. The area where forward link power is below tag sensitivity and/or backscattered link power is below reader sensitivity is shaded in black. The results indicates that when $K_{f}$ and $K_{b}$ are small, there is a severe multipath fading effect which makes the reliable reading zone more difficult to reach when tags are further away from the transmitting antenna. In addition, there is a strong fluctuation in the received RSSI when tags are close to the ground. This is expected since tags closer to the ground are more subject to ground reflection.

Figure 4 illustrates how the tag detection probability varies with average received power for different values of $K$-factor

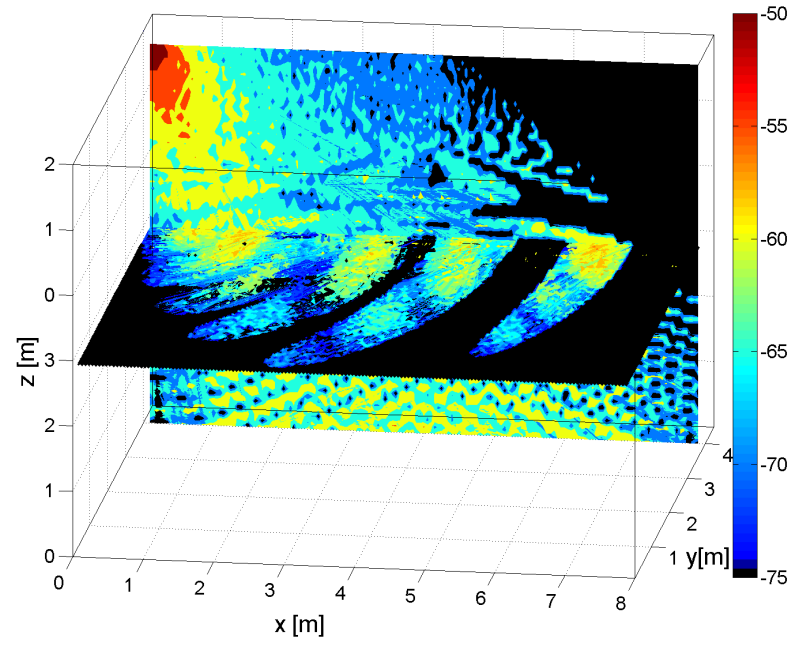

Fig. 3. 3D view of passive RFID RSSI distribution for $K_{f}=K_{b}=0 \mathrm{~dB}$, $\theta_{f}=\theta_{b}=\pi / 4$ and based on bistatic two rays model.

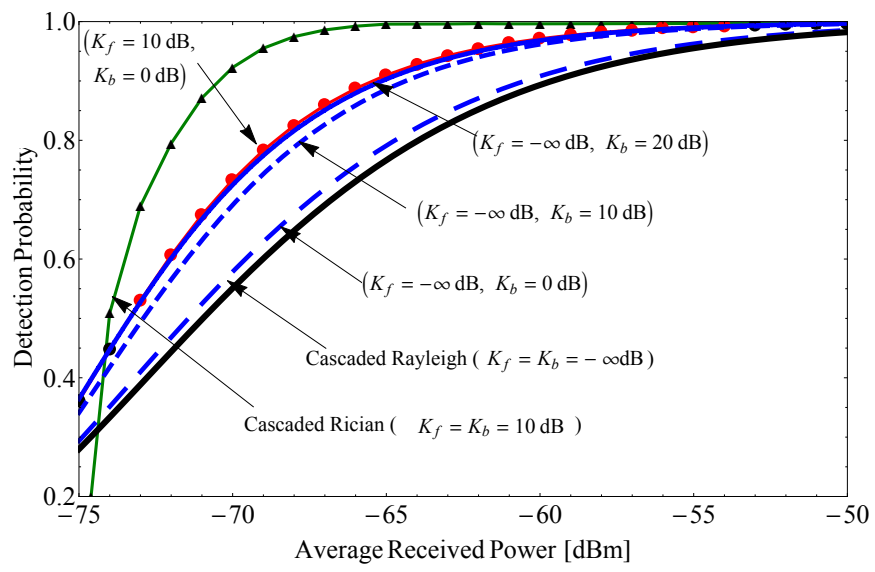

Fig. 4. Detection probability as a function of average received power for different $K$-factor values.

for forward and backscatter links. As expected, the tag detection probability is lowest when $K_{f}=K_{b}=-\infty \mathrm{dB}$ (cascaded Rayleigh) due to the absence of the LOS in both forward and backscattered link and it is the highest when $K_{f}=K_{b}=20 \mathrm{~dB}$ (cascaded Rician with strong LOS environment). For instance, when $K_{b}=-\infty \mathrm{dB}$ and $K_{f}$ increases, the forward link is exposed to stronger LOS environment, and the tag detection probability is increased accordingly. However, the tag detection probability begins to saturate when $K_{f}$ reaches $20 \mathrm{~dB}$ (almost no fading) and further increasing $K_{f}$ does not provide extra benefits in the tag detection probability. Hence, the maximum benefits on tag detection probability brought by increasing $K$-factor is around $10 \%$ on average. After this point, detection probability is mainly dominated by Rayleigh faded backscattered link, which results in reduced detection probability even when the average received power is well above reader sensitivity. 


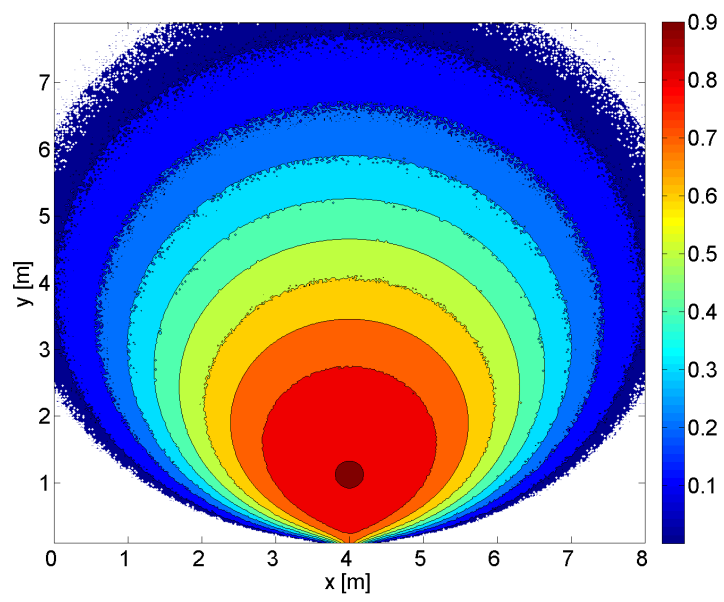

(a)

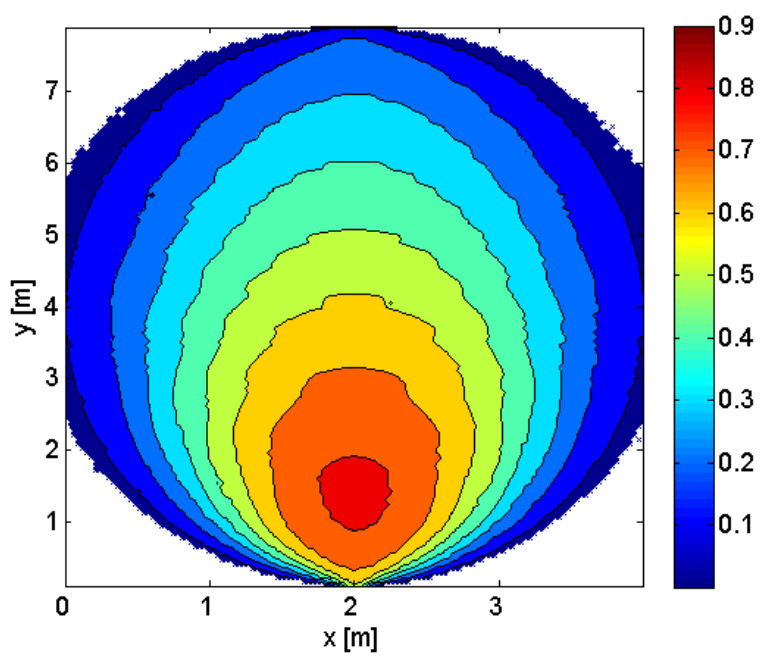

(b)

Fig. 5. 2-D distribution of the detection probability for tags located on the same level of $1.5 \mathrm{~m}, K_{f}=K_{b}=-\infty \mathrm{dB}, \theta_{f}=\theta_{b}=\pi / 6$ and using two rays model: (a) Monostatic configuration, (b)Bistatic configuration.

To illustrate the difference between the monostatic and bistatic reading coverage, we choose the worst scenario which is double Rayleigh (i.e. $K_{f}=K_{b}=-\infty \mathrm{dB}$ ). As can be seen from Fig.5, the two systems exhibits similar envelopes of detection region due to tag sensitivity constraints in the forward link power. However, the distribution of tag detection probability between these two systems is quite different. In monostatic case tags have high detection probability when they are close to the reader antenna, whereas in bistatic system tags are more likely to be detected when they are closer to the center area of the transmitting and receiving antenna. This suggests that bistatic system has more directional distribution of tag RSSI in the tag plane and therefore might enable larger reliable reading range compared to monostatic system if properly configured.

To show the impact of fading on the variation of detection probability for the case of bistatic scenario, Fig. 6 plots the
2-D detection probability distribution of a tag on the plane level with $H=1.5 \mathrm{~m}$, for different forward and backscattered link fading parameters. Generally, we can see that the reliable reading zone increases from the case of Rician faded forward link to Rayleigh faded backscattered link to double Rician case. The reliable reading zone also increases drastically and becomes more well defined with increasing $K$-Factor. This is because when $\mathrm{K}$ factors are increased, multipath effect is effectively reduced and hence phase and amplitude uncertainty. It is clear that the double Rician case with the highest $K_{f}$ and $K_{b}$ factors, Fig. 6(d) represents a nearly ideal environment and therefore, a clear and sharp edge in detection probability is seen when backscattered RSSI is below reader sensitivity.

Next, in order to study the effect of interference on the passive RFID performance, we consider that the passive RFID link is FLL where the maximum reading range is limited by the tag sensitivity. The numerical results of the tags detection probability and reader reading coverage are generated and studied based on equations Eq.1 and Eq.22.

To highlight the effect of constructive and destructive interference presented in section IV.B, Fig. 7 illustrates the distribution of the field strength and the occurrence of constructive and destructive areas for two RFID antennas when Rician K-Factor $K_{f}=0 \mathrm{~dB}$. The result shows that when multiple antennas are used to transmit identical signals simultaneously the reading range is extended, although there will be large power variation in the interrogation area due to constructive and destructive interference. The destructive interference is particularly harmful to forward link reader-to-tag communication because of poor tag sensitivity of passive RFID tags. Tags in such destructive area might receive insufficient power to activate their internal circuit and hence, undetectable by the reader even well within the interrogation range. This suggests that more intelligent techniques should be used in multiple input multiple output (MIMO) RFID system such as phase dithering to effectively move destructive interference around and eliminate tag nulls [31].

In the following and without loss of generality, we assume that the distance $H$ between tag location and its orthogonal projection on the reader plane $(x, y)$ equals to 0 (i.e. $H=0$ ) and hence, the readers antennas will be facing each others with a relative orientation $\theta=\left|\theta_{D}-\theta_{I}\right|$, where $\theta_{D}$ and $\theta_{I}$ are the desired and interfering reader antenna elevation angle, respectively.

Next, we analyze the impact of tag jamming which is a type of destructive interference occurring between signals from different RFID readers as explained in section IV.A.2. In Fig. 8, the variation of the tag detection probability with respect to the reader-to-tag distance for different values of Rician K-Factors $\left(K_{0}, K_{I}\right)=(0 \mathrm{~dB}, 0 \mathrm{~dB}),(10 \mathrm{~dB}, 10 \mathrm{~dB}),(20 \mathrm{~dB}, 20 \mathrm{~dB})$ and with readers separation distance $d_{D R-I R}=10 \mathrm{~m}$ are depicted. As can be seen from the figure, the detection probability drops when the tag is further away from the desired reader. In addition, the severe multipath (i.e. $K_{0}=K_{I}=$ $0 \mathrm{~dB}$ ) can also introduce reliable reading range penalty due to large forward link power variance in the interrogation zone. It is also interesting to notice that beyond a certain range (detection probability $<50 \%$ ) the multipath effect actually 


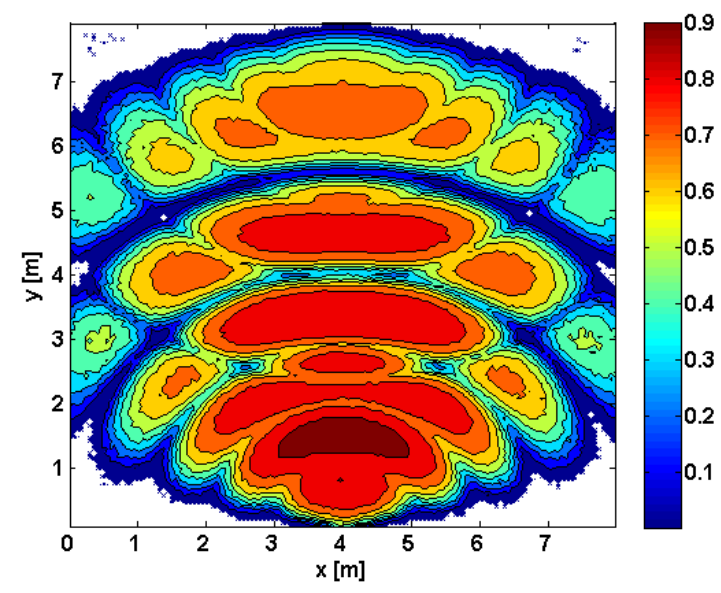

(a)

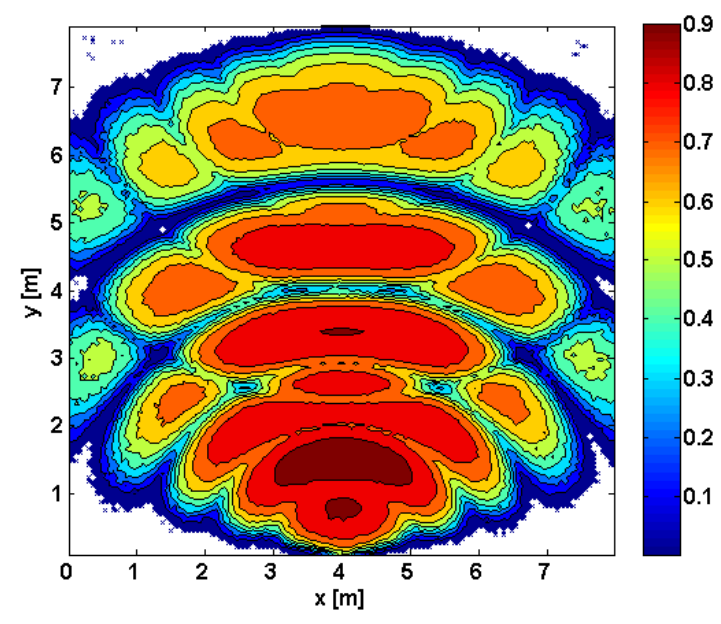

(c)

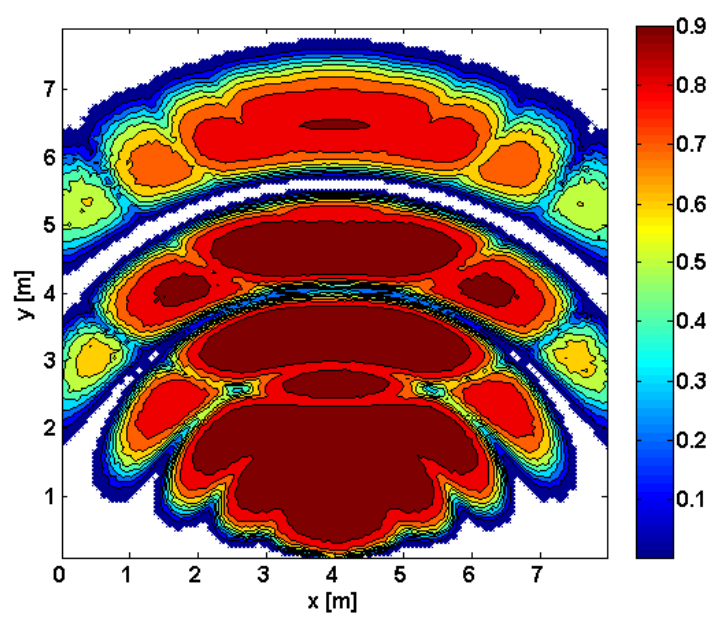

(b)

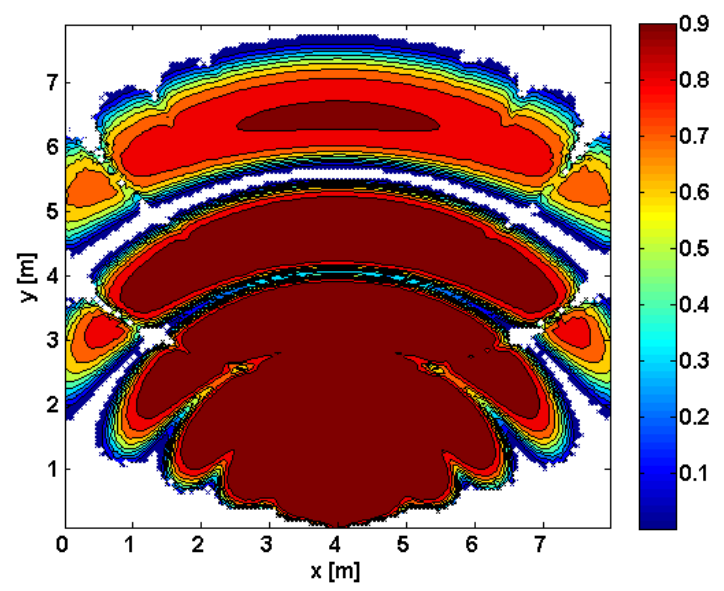

(d)

Fig. 6. 2D-distribution of the detection probability for tags located on the same level of $1.5 \mathrm{~m}, \theta_{f}=\theta_{b}=\pi / 6$ and using bistatic two rays model (a) $K_{f}=0 \mathrm{~dB}, K_{b}=-\infty \mathrm{dB}$, (b) $K_{f}=10 \mathrm{~dB}, K_{b}=-\infty \mathrm{dB}$, (c) $K_{f}=K_{b}=0 \mathrm{~dB}$, (d) $K_{f}=K_{b}=10 \mathrm{~dB}$.

boosts detection probability compared to nearly free space (i.e $K_{0}=K_{I}=20 \mathrm{~dB}$ ). This is because the power received beyond this range is weak and the power variance due to multipath effect may contribute constructively in increasing the forward link power.

To assess the impact of the desired reader-tag misalignment angle (i.e. the angle between the main lobe of an antenna and its direction to the tag) on the reading reliability, we plot in Fig. 9 the reliable reading range as a function of the misalignment angle ranging from $-\pi / 2$ to $\pi / 2$, for $K_{0}=K_{I}=20 \mathrm{~dB}$, the inclination angle of the interferer antenna $\theta_{I}=0$, (i.e. maximum transmission gain to the tag) and different values of SIR threshold $\operatorname{SIR}_{\mathrm{th}}=\{0 \mathrm{~dB}, 5 \mathrm{~dB}, 10 \mathrm{~dB}\}$. The reliable range is represented as a percentage of the separation distance between the desired and interfering reader $d_{I}$. When the desired reader and tag antennas are perfectly aligned, the received power is maximized, which in turn maximizes the reliable reading range. As the tag and desired reader become misaligned, the performance is reduced due to the reduction of the received power from the desired reader relative to the received power from the interferer. It can be seen that misalignment angle has a huge impact on the reliable reading range. For instance, when $\mathrm{SIR}_{\mathrm{th}}=0 \mathrm{~dB}$ and separation distance $d_{I}=10 \mathrm{~m}$, the reliable range penalty is almost $3.5 \mathrm{rad}$ (or $0.06 \mathrm{~m} / \mathrm{deg}$ ).

Let us now analyze the effect of the separation distance between the desired reader and the interfering reader $d_{I}$ on the reliable reading range. Fig. 10 plots the variation of the reliable reading range with respect to $d_{I}$, for different number of interferers $N_{I}=1,2,3,4, K_{0}=K_{I}=20 \mathrm{~dB}$ and $\operatorname{SIR}_{\text {th }}=$ $10 \mathrm{~dB}$. From the figure, It can be noticed that when the separation distance $d_{I}$ increases, a roughly linear effect is seen on the increase of reliable reading range. This is due to the tag jamming that happens when the tag is located in a the common interrogation area of both readers. If the readers are far enough apart, the tag jamming zone lies outside of the reading range of the tag. On the other hand, if they are close to each other, the tag jamming zone can fall inside a reading zone and its area increases as the separation distance $d_{I}$ decreases. Tag 


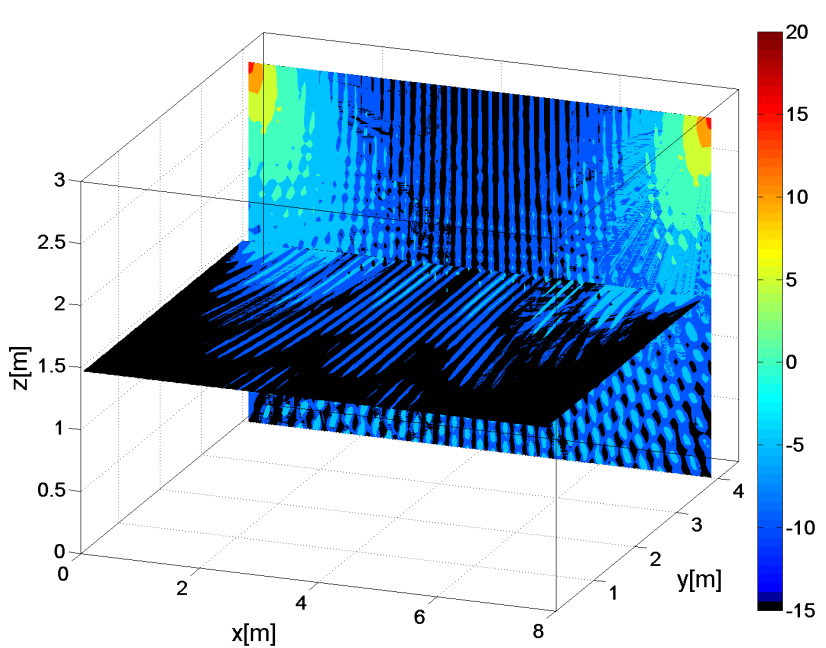

Fig. 7. 3-D View of two readers constructive interference with $K=0 \mathrm{~dB}$.

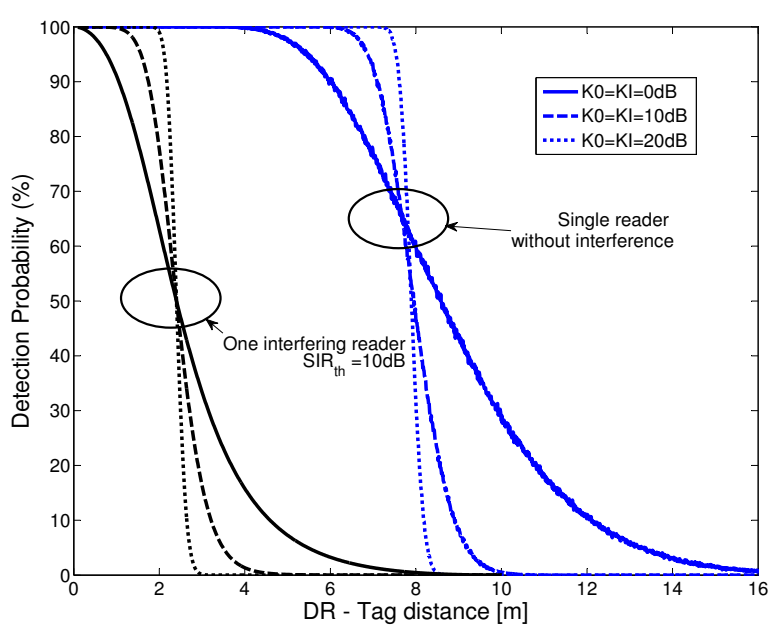

Fig. 8. Effect of Tag Jamming for different multipath environments, $\mathrm{SIR}_{\mathrm{th}}=$ $10 \mathrm{~dB}$ and Readers separation distance of $10 \mathrm{~m}$.

jamming is primarily related to transmission power levels and the proximity of readers. However, the reliable reading range cannot increase indefinitely and is subject to a threshold power to activate the internal circuits of a tag (i.e. tag sensitivity). Therefore a saturation effect is observed when $N_{I}=1$. Furthermore, increasing the number of interfering readers also leads to a reduction of the reliable reading range. To highlight more the impact of the number of interfering readers, Fig. 11 plots the variation of reliable reading range with respect to the number of interfering readers for different SIR threshold level $\mathrm{SIR}_{\mathrm{th}}=\{0 \mathrm{~dB}, 5 \mathrm{~dB}, 10 \mathrm{~dB}\}$. As expected, the reliable reading range drops drastically when the number of interfering readers increases. For instance, when the $\mathrm{SIR}_{\text {th }}=5 \mathrm{~dB}$ the reliable reading range drops from $8 \mathrm{~m}$ for the case of without

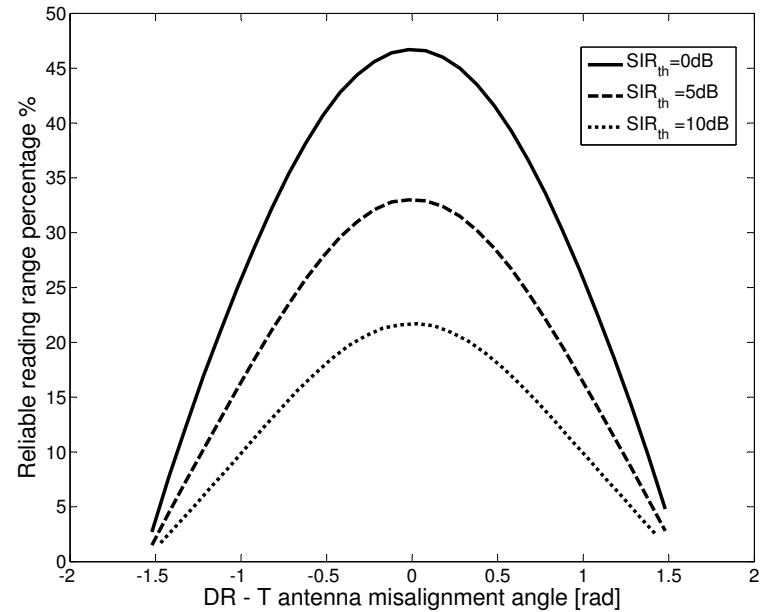

Fig. 9. Reliable reading range for reader-tag misalignment angle for $K_{0}=$ $K_{I}=20 \mathrm{~dB}$ and different value of $\mathrm{SIR}_{\mathrm{th}}=0 \mathrm{~dB}, 5 \mathrm{~dB}$, and $10 \mathrm{~dB}$.

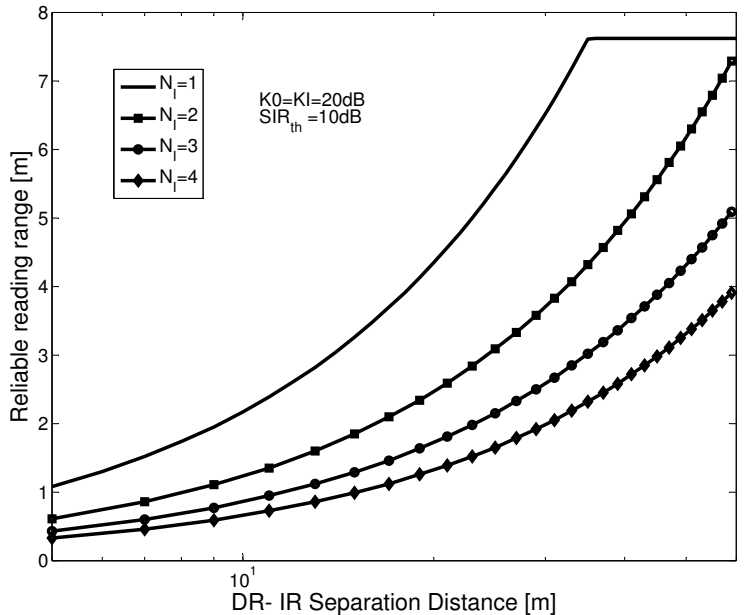

Fig. 10. Reliable reading range vs. readers separation distance with $\mathrm{SIR}_{\mathrm{th}}=$ $10 \mathrm{~dB}$ and $K_{0}=K_{I}=20 \mathrm{~dB}$.

interference $\left(N_{I}=0\right)$ to about $2 \mathrm{~m}$ when the number of interfering readers increases by $2\left(N_{I}=2\right)$.

\section{CONCLUSION}

In this paper, we have studied the performance of passive RFID system under constructive and destructive interference and cascaded channel fading from the RF point of view. We have presented a 3-D analytical model for RFID system and studied the effect of cascaded channel fading and interference, in terms of detection probability. We have derived a closedform expression for passive RFID detection probability taking into account the relative reader-tag antennas orientations and Rician/Rayleigh cascaded channel fading parameters. We have also analyzed the tag detection probability at different 


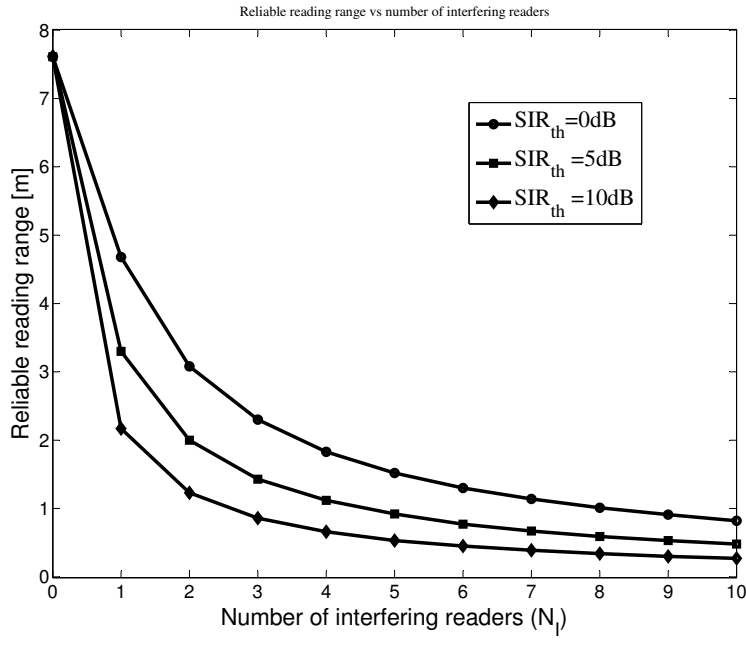

Fig. 11. Effect of number of interfering readers for different value of $\mathrm{SIR}_{\mathrm{th}}=$ $0 \mathrm{~dB}, 5 \mathrm{~dB}$, and $10 \mathrm{~dB}$ and $K_{0}=K_{1}=20 \mathrm{~dB}$.

interference environment and fading levels modeled by Rician and Rayleigh distribution. In contrast to the conventional RF communication links, our results show that the passive RFID system performance is highly sensitive to the interference and channel fading and thus for a maximum reliable reading range (i.e. $100 \%$ successful detection probability), proper conditions should be analyzed and defined before any implementation of the RFID system.

\section{REFERENCES}

[1] K. Finkenzeller. "RFID Handbook: Radio-Frequency Identification Fundamentals and Applications". John Wiley \& Sons, 2000.

[2] A. Bekkali and M. Matsumoto, "Bayesian sensor model for indoor localization in ubiquitous sensor network, "in Proc. 1st ITU-T Kaleidoscope Conference, 2008, pp. 285-292.

[3] R. Want, "An introduction to RFID technology". IEEE Pervasive Computing, 5(1):25-33, Jan.-March

[4] "RFID communication and interference", White Paper, Grand Prix Application Series, Impinj. Inc, 2007. 2006.

[5] J. D. Griffin and G. D. Durgin "Complete link budgets for backscatterradio and RFID systems", IEEE Antennas Propag. Mag., vol. 51, no. 2, pp.11 -25 2009.

[6] V. Pillai, "Impedance matching in RFID tags: to which impedance to match ?," in IEEE AP-S International Symposium., pp.3505-3508, 2006.

[7] D. Kim, M. A. Ingram, and W. W. Smith, Jr., "Measurements of Smallscale Fading and Path Loss for Long Range RF Tags,"IEEE Transactions on Antennas and Propagation, AP-51, 8, 2003, pp. 1740-1749.

[8] C. H. Loo, A. Z. Elsherbeni, F. Yang, and D. Kajfez, "Experimental and Simulation Investigation of RFID Blind Spots," Journal of Electromagnetic. Waves and Applications, Vol. 23, 747-760, 2009.

[9] P. V. Nikitin and K. V. S. Rao, "Performance limitations of passive UHF RFID systems", IEEE Antennas and Propagation Symposium, pp. 10111014, 2006.

[10] A. Lazaro, D. Girbau, D. Salinas. "Radio link budgets for UHF RFID on multipath environments". IEEE Transactions on Antennas and Propagation, 2009, vol. 57, no. 4, p. 1241 - 1251.

[11] J. D. Griffin and G. D. Durgin "Gains for RF tags using multiple antennas". IEEE Transactions on Antennas and Propagation, 2008, vol. 56 , no 2 , p. $563-570$.

[12] D.-Y. Kim, H.-S. Jo, H. Yoon, C. Mun, B.-J. Jang, and J.-G. Yook. "Reverse-link interrogation range of a UHF MIMO-RFID system in Nakagami-m fading channels". IEEE Trans. Industrial Electronics, 57:1468-1477, 2010.
[13] D.-Y. Kim, H.-G. Yoon, B.-J. Jang, and J.-G. Yook, "Effects of reader interference on the RFID interrogation range," IEEE Trans. Ind. Electron., vol. 56, no. 7, pp. 2337-2346, Jul. 2009.

[14] A. Lazaro, D. Girbau, and R. Villarino, " Effects of interferences in UHF RFID systems,"Progress In Electromagnetics Research, Vol. 98, 425-443, 2009.

[15] A. G. Dimitriou, A. Bletsas, J. N. Sahalos, "Room Coverage Improvements in UHF RFID with Commodity Hard ware, "IEEE Antenna and Propagation Magazine, 53, 1, 2011, pp. 175-194.

[16] G. Marrocco, E. Di Giampaolo, R. Aliberti "Estimation of UHF RFID reading regions in real environments". IEEE Antennas and Propagation Magazine, vol. 51, no 6, p. 44 - 57, 2009.

[17] S. R. Aroor and D. D. Deavours, "Evaluation of the state of passive UHF RFID: an experimental approach", IEEE Systems Journal, vol. 1, no. 2, pp. 168-176, 2007.

[18] T. Rappaport “Wireless Communications - Principles and Practice. IEEE Press 1996.

[19] J.S. Colburn, M.A. Jensen, Y. Rahmat-Samii, "Indoor ISM Band Multipath Fading: Frequency and Antenna Diversity, "IEEE APS Conference on Antennas and Propagation for Wireless Communications, pp: 9-12, 1998.

[20] E. Greene, "Area of Operation for Radio-Frequency Identification (RFID) Tag in the Far-Field ", PhD Thesis, Univ. of Pittsburgh, Pittsburgh, Feb. 2006.

[21] EPC Radio-Frequency Identity Protocols Class-1Generation-2 UHF RFID Protocol for Communications at $860 \mathrm{MHz}-960 \mathrm{MHz}$ Version 1.0.9," EPC Global, Tech. Rep., Jan 2005.

[22] M. K. Simon, "Probability Distributions Involving Gaussian Random Variables: A Handbook for Engineers and Scientists "Norwell, MA: Kluwer Academic, 2002.

[23] I. Gradsheteyn and I. Ryzhik,"Table of Integrals, Series and Product "7th ed., New York: Academic Press, 1994.

[24] ETSI EN 302 208-1 V1.1.1.

[25] K. S. Leong, M. L. Ng, Alo R. Grasso ,P. H. Cole, "Dense RFID Reader Deployment in Europe using Synchronization", Journal of Communications, Vol. 1, No. 7, Dec 2006.

[26] S. Jain, S. Das "Collision Avoidance in a Dense RFID Network", WiNTECH06, September 29, 2006, Los Angeles, California, USA.

[27] D. W. Engels, "The Reader Collision Problem,"MIT Auto ID Center, MIT-AUTOID-WH-007, 2002.

[28] T. T. Tjhung, C. C. Chai, and X. Dong, "Outage probability for lognormal-shadowed Rician channels," IEEE Trans. Veh. Technol., vol.46, pp. 400-407, May 1997.

[29] P.V. Nikitin and K.V.S. Rao, "Effect of gen2 protocol parameters on RFID tag performance"in Proc. 2009 Intl. Conf. on RFID, pp.117-122, Apr.2009.

[30] A. G. Dimitriou, A. Bletsa, A.C. Polycarpou, J. N. Sahalos "Theoretical Findings and Measurements on Planning a UHF RFID System inside a Room" Radioengineering, Vol. 20, No. 2, JUNE 2011.

[31] S. Sabesan, M.J. Crisp, R.V. Penty, I.H. White, "Wide Area Passive UHF RFID System Using Antenna Diversity Combined With Phase and Frequency Hopping,"IEEE Transactions on Antennas and Propagation, vol.62, no.2, pp.878,888, Feb. 2014 